\title{
Early childhood caries
}

\author{
Abstracted from
}

Leong PM, Gussy MG, Barrow SY, de Silva-Sanigorski A, Waters E.

A systematic review of risk factors during first year of life for early childhood caries. Int J Paediatr Dent. 2012; Aug 28. doi: 10.1111/j.1365-263X.2002.01260.x

Address for correspondence: PM Leong, Department of Dentistry, Royal Children's Hospital, Parkville, PO Box 9403, South Yarra, Vic., 3141, Australia. E-mail: pamandyin@gmail.com

\section{Question: What factors during the first year of an infant's life influence the initiation and progression of early childhood caries?}

Data sources Medline and Embase electronic databases were searched.

Study selection Papers published between 1996 and 2011 were initially identified by one reviewer, with $10 \%$ being independently reviewed by a second reviewer having predefined inclusion and exclusion criteria.

Data extraction and synthesis Data abstraction was conducted independently and meta-analysis was not attempted because of the heterogeneity of the studies.

Results Inclusion criteria were met by four papers relating to the acquisition and colonisation of the oral cariogenic bacteria and caries outcome in infants, 13 papers were considered in relation to identifying possible determinants of early childhood caries (ECC) during the first year of life.

Conclusions The review confirmed that factors occurring during the first year of life affect ECC experience. Despite heterogeneity, findings indicated maternal factors influence bacterial acquisition, whereas colonisation was mediated by oral health behaviour and practices and feeding habits.

\section{Commentary}

Early childhood caries is defined as the presence of decay in one or more primary teeth in a preschool child between birth and 71 months. ${ }^{1}$ Prevalence for ECC ranges from $28 \%$ to $82 \%$ depending on the population studied; these figures therefore suggest that ECC is a widespread public health concern. ECC is also a strong predictor of dental caries in later life and is largely preventable.

Pursuing answers to the question 'what influences ECC?' is therefore important and fundamental in the aetiology of dental disease and would help develop public health policy and appropriate oral health promotion and prevention programmes. It is evident that ECC is multi-factorial and further research is required to identify the key risk factors and an effective evidence-based strategy to screen infants in order to help identify those at risk of ECC and ensure an appropriate level of care is provided. ${ }^{2}$

The objective of this review was to evaluate the available evidence on the risk factors during the first year of life for early childhood caries as measured by mutans streptococci (MS) testing and considering the risk and protective factors for ECC.

Previous reviews have focused on specific risk factors; a systematic review conducted by Parisotto et al. $(2010)^{3}$ looked at MS and included 16 studies. They concluded that MS was a strong risk indicator for ECC. In 2004, Harris et al. ${ }^{4}$ looked at risk factors for dental caries in young children and identified over 100 potential risk factors. Harris et al. grouped the risk factors into six main categories - socio-demographic, dietary, oral hygiene, factors relating to breast or bottle feeding, oral bacterial flora and other factors, and looked at the risks and compensating factors such as diet and oral hygiene.

Meaningful research considering the risk assessment for ECC to date suggests few definitive conclusions and highlights the challenges of undertaking cariological bacterial assessments on children under one year old - this is a challenging group to conduct research on because of other competing priorities and as such it can be difficult to influence or change behaviours.

This review had two separate objectives that were more weighted towards MS as opposed to other risk factors, leading to an inherent level of bias. Within the narrative the inclusion and exclusion criteria were ill defined, which could result in the misinterpretation of the flow diagrams. Two separate search strings were identified for the two objectives. A different approach could have been undertaken to search the literature addressing the overall aim of the study, prior to refining the searches for further sub-group analysis.

Two databases were searched and no language restrictions were applied, but as translators were not available for the relevant papers, 
personal communication was made with authors for additional clarification. Additional databases (eg LILACS or Chinese biomedical database) may have produced additional papers. The identified studies spanned from 1996-2010 and it could be questioned why 1996 was set for both components of the research - perhaps it is justifiable for the MS and scientific testing but could have been extended to include further studies on the risk factors.

The quality of the studies was assessed using the CASP (Critical Appraisal Skills Programme)(www.casp-uk.net), which has a suite of tools for different types of study design; whilst this is a good tool for critically appraising papers, it is unclear as to the methodology used to score the included papers. Other more relevant quality assessment tools could have been used such as the Newcastle-Ottawa Scale (NOS). The NOS is available to assess the quality of non-randomised studies for systematic reviews.

Prisma-like flow charts (www.prisma-statement.org) were provided and offer some clarification to the reader as to the reasons for exclusion; due to the two questions being addressed there is some confusion as to the number of studies mentioned in the text and the numbers included in the flow charts, which detracts from the overall review.

Four papers met the inclusion criteria for the first objective and 13 papers were included for the second objective, three papers were common in both searches.

Due to the heterogeneity of the studies and the variation in the quality and presentation of the results meta-analysis was not attempted. Study designs included randomised control trials, longitudinal cohort studies and cross-sectional studies. There were variations in the methods used for collecting and measuring MS, and different sites within the mouth were used which could have further influenced the level of MS recorded - all these factors led to non-homogeneous studies.

A lengthy descriptive summary of the findings was given making the overall results difficult to find. Interestingly, in three studies MS was found in the mouth of some infants before the eruption of the first tooth - previous research has suggested MS was present only when teeth were present. Harris et al. ${ }^{4}$ also noted in their systematic review that MS was generally only detectable in the presence of teeth, and the age at which MS is acquired is a significant risk factor in the development of ECC. Another point of interest is that not all children harbouring MS go on to develop caries and this requires further research.

The review did not show conclusive evidence that spending resources to screen for MS was beneficial and influenced the outcome or dentists' behaviours in providing dental prevention. Social factors and other risk factors, based on the residential postcode, previous caries experience and siblings' experience of dental caries aid the decision making process during the dentist's treatment plan.

\section{Conclusion}

Maternal factors and oral health behaviours affect the acquisition and colonisation of MS and ECC. Further work is required on this important topic, including the exploration of the recently published Dundee Caries Risk Assessment Model (DCRAM), ${ }^{5}$ but the key messages include the practice points below to help prevent ECC and potential dental caries. Current evidence does not support the routine use of MS testing, as not everyone with MS goes on to develop ECC although Yoon et al.'s ${ }^{2}$ work shows using MS cultures and variations on a Caries-risk Assessment Tool (CAT) are 'promising approaches for identifying children who need early and intensive treatment to prevent or minimise caries experience'. Other risk factors are involved in the development of ECC and engaging in ECC studies and MS testing might raise the issue of maternal and infant oral health sufficiently and influence a shift in oral health practice.

\section{Practice point}

- Work with healthcare professionals to promote the common risk approach and ensure a consistent message is delivered in relation to dietary advice and oral health education

- Engage with pregnant women and mothers to ensure their own oral health is maintained

- Promote dental registration from birth

- Educate parents and caregivers about reducing the risks for ECC

- Recommend that the introduction of complementary foods (weaning) is around six months for all infants. ${ }^{6}$

\section{Emma O'Keefe}

Department of Public Health, NHS Fife, Leven, Scotland, UK.

1 American Academy of Pediatric Dentistry, American Academy of Pediatrics. Policy on early childhood caries (ECC): classifications, consequences, and preventive strategies. Pediatr Dent. 2008-2009; 30: 40-43.

2 Yoon RK, Smaldone AM, Edelstein BL. Early childhood caries screening tools: a comparison of four approaches. / Am Dent Assoc. 2012; 143: 756-763.

3 Parisotto TM, Steiner-Oliveira C, Silva CM, Rodrigues LK, Nobre-dos-Santos M. Early childhood caries and mutans streptococci: a systematic review. Oral Health Prev Dent. 2010; 8: 59-70.

4 Harris R, Nicoll AD, Adair PM, Pine CM. Risk factors for dental caries in young children: a systematic review of the literature. Community Dent Health. 2004; 21: 71-85.

5 MacRitchie HM, Longbottom C, Robertson M, Nugent Z, Chan K, Radford JR, Pitts NB. Development of the Dundee Caries Risk Assessment Model (DCRAM) - risk model development using a novel application of CHAID analysis. Community Dent Oral Epidemiol. 2012; 40: 37-45.

6 NHS Health Scotland. Oral Health and Nutrition Guidance for Professionals. Edinburgh: NHS Health Scotland 2012.

Evidence-Based Dentistry (2013) 14, 40-41. doi:10.1038/sj.ebd.6400928 